\title{
Iterative Power Control and Multiuser Detection with Outage Probability Constraints
}

\author{
John Papandriopoulos, Jamie Evans and Subhrakanti Dey \\ ARC Special Research Centre for Ultra-Broadband Information Networks (CUBIN), \\ Department of Electrical and Electronic Engineering, \\ University of Melbourne, VIC 3010, Australia
}

\begin{abstract}
This paper proposes a new scheme coupling power control with a minimum outage probability multiuser detector. The resultant iterative algorithm is conceptually simple and finds the minimum sum transmission power of all users with a set of outage probability constraints. Bounds on the outage probability expression are found that extend a previous result that did not include receiver noise. These bounds are used to create a suboptimal scheme coupling power control and a MMSE multiuser detector. This new problem becomes a variant of an existing problem where outage probability constraints are first mapped to average SIR threshold constraints. Simulation results are presented showing the closeness of the two schemes and speed of convergence.
\end{abstract}

\section{INTRODUCTION}

Power allocation is an effective way to improve the performance of wireless communication systems. It can mitigate the near-far problem occurring when a nearby interferer disturbs the reception of a remote user, whose desired signal is attenuated to a greater extent. Careful power allocation can also increase utilization in interference-limited systems such as CDMA, or those multiple access systems employing frequency reuse amongst cells, as in FDMA. Finally, by allocating minimum power across all users, battery life of mobile devices will be extended: users only need to expend sufficient power for acceptable reception as determined by their quality of service (QoS) specifications such as FER, BER or outage probability.

The power allocation problem has been studied extensively as an eigenvalue problem for non-negative matrices [1]-[4], as iterative Power Control Algorithms (PCA) that converge each user's power to the minimum power result [5]-[6], optimization based approaches [7]-[9] and other variations [10]-[12]. A useful framework for uplink cellular power control is given in [13].

Much of this previous work deals with invariant channel models. Any power control scheme that attempts to follow fast fades would need to be highly efficient to be implemented in practice, or incur a power penalty due to intense signal processing and may require frequent communication with its assigned base station.

Of particular interest is the work presented in [8] and [9]. In [8], a scheme whereby the statistics of the received signal to noise ratio (SIR) are used to allocate power, rather than an

This work was supported by the Australian Research Council. instantaneous SIR. The allocation decisions can then be made on a much slower time scale (following log-normal shadowing variations for instance). In [9], an optimization problem is considered using average SIR which yields locally optimal solutions.

In a multiple access system, multiuser detection (MUD) can be used to further enhance the performance of wireless systems by exploiting the structure of the multiple access interference [14]. The optimal MUD is near-far resistant however has exponential complexity with the number of active users. The decorrelating detector, with polynomial complexity, can eliminate multiuser interference entirely at the expense of enhancing receiver noise power [15]. Furthermore, in a CDMA system, the noise enhancement increases as the utilization increases until the detector becomes unusable. Minimum mean squared error (MMSE) detection [16] is based on the minimization of the expected squared error between a transmitted symbol and the received signal. It is also near-far resistant and reduces to the decorrelating detector as the AWGN power is zeroed. The MMSE receiver is considered a compromise between the matched filter and decorrelator.

Traditionally, power allocation and multiuser detection were considered separately. Power allocation assumed a fixed receiver structure and multiuser detection assumed fixed user transmitter powers. Recent work [17]-[19] has focused on the problem of jointly optimizing both power and linear receivers to obtain the benefits from both power allocation and MUD.

The main contributions of this paper are:

1) Design of a conceptually simple, iterative algorithm that minimizes the sum power of all users subject to outage constraints. The optimization is performed on choice of user powers and linear multiuser receivers. This problem reduces to that considered in [8] if background noise is neglected and linear receivers fixed.

2) A new sub-optimal iterative PCA-MUD where outage constraints are first mapped to average SIR threshold constraints. Such a mapping permits the use of a variant of an existing combined PCA and MMSE MUD given in [17].

Simulation results are also provided comparing both methods.

\section{System Model}

In this paper, we consider the uplink in a synchronous direct sequence CDMA communications system with $K$ users 
and a processing gain of $N$. We assume a BPSK modulation scheme and a $N$-dimensional chip matched filter vector for each symbol interval, given by

$$
\mathbf{r}_{i}=\sum_{j=1}^{K} \sqrt{G_{i j} F_{i j} P_{j}} b_{j} \mathbf{s}_{j}+\mathbf{n}
$$

where $G_{i j} F_{i j} P_{j}$ is the total received power from user $j$, with transmit power $P_{j} . G_{i j} F_{i j}$ represents the instantaneous channel gain of user $j$ to the assigned Base Station (BS) of user $i$. The data bits $b_{j}$ take on values of \pm 1 with equal probability, $\mathbf{s}_{j}$ is the $N$-dimensional spreading sequence of user $j$, and $\mathbf{n}$ is AWGN with zero mean and covariance $\sigma^{2} \mathbf{I}$. We assume fixed spreading sequences, with elements of $\mathbf{s}_{j}$ taking values $\pm 1 / \sqrt{N}$.

Following the conventions of [8], we assume $G_{i j}$ is the positive slow-varying path gain of user $j$ to the assigned Base Station (BS) of user $i$, excluding any fading. The analysis that follows holds only over a time scale where factors affecting $G_{i j}$ do not change significantly. An adaptive channel estimator can be utilized to reassess the allocation of powers modifying the following work to create an adaptive PCA.

The terms $F_{i j}$ model fast time scale Rayleigh fading and are assumed to be unit mean independent exponentially distributed random variables. In this so called Rayleigh/Rayleigh fading environment, the received power has mean value

$$
E\left[G_{i j} F_{i j} P_{j}\right]=G_{i j} P_{j}
$$

Let $\mathbf{c}_{i}$ denote the receiver filter coefficients for user $i$ at its assigned $\mathrm{BS}$ and $\mathbf{c}=\left[\mathbf{c}_{1}, \ldots, \mathbf{c}_{K}\right]$. The filter output of user $i$ at its assigned $\mathrm{BS}$ is given by

$$
\mathbf{y}_{i}=\mathbf{c}_{i}^{\top} \mathbf{r}_{i}=\sum_{j=1}^{K} \sqrt{G_{i j} F_{i j} P_{j}}\left(\mathbf{c}_{i}^{\top} \mathbf{s}_{j}\right) b_{j}+\tilde{n}_{i}
$$

where $\tilde{n}_{i}=\mathbf{c}_{i}^{\top} \mathbf{n}$ is $N\left(0, \sigma^{2} \mathbf{c}_{i}^{\top} \mathbf{c}_{i}\right)$.

Unlike the development in [8], we will not neglect receiver noise as it will be crucial to the PCA developed below.

\section{Outage Probability And Certainty-EQuivalent MARGIN WITH NOISE}

To simplify notation in this section, we shall drop the receiver filter terms $\left(\mathbf{c}_{i}^{\top} \mathbf{s}_{j}\right)^{2}$ without a loss of generality (since we can absorb them into the $G_{i j}$ terms). They will be important in Section IV.

\section{A. SIR and Outage Probability}

The SIR, $\gamma$, of the $i$ th mobile is given by

$$
\gamma_{i}=\frac{G_{i i} F_{i i} P_{i}}{\sum_{j \neq i} G_{i j} F_{i j} P_{j}+\sigma^{2}} .
$$

The outage probability of user $i$, denoted $O_{i}$, is defined as the proportion of time that some SIR threshold $\gamma_{i}^{t h}$ is not met for sufficient reception at the BS receiver. By careful choice of $\gamma_{i}^{t h}$, we can set a QoS for each user. $O_{i}$ is given by

$$
\begin{aligned}
O_{i} & =\operatorname{Pr}\left(\gamma_{i} \leq \gamma_{i}^{t h}\right) \\
& =\operatorname{Pr}\left(G_{i i} F_{i i} P_{i} \leq \gamma_{i}^{t h}\left\{\sum_{j \neq i} G_{i j} F_{i j} P_{j}+\sigma^{2}\right\}\right) .
\end{aligned}
$$

The outage probability for the $i$ th user is given by (see [8])

$$
O_{i}=1-\exp \left(\frac{-\sigma^{2} \gamma_{i}^{t h}}{G_{i i} P_{i}}\right) \prod_{j \neq i} \frac{1}{1+\frac{\gamma_{i}^{t h} G_{i j} P_{j}}{G_{i i} P_{i}}} .
$$

\section{B. Certainty-Equivalent Margin with Noise}

The Certainty-Equivalent Margin (CEM) was defined in [8] without noise. It represents a margin of error for average SIR when representing the system by a certainty-equivalent form (with all statistical variation in signal and noise power ignored and replaced with their expected values.)

We will take average SIR ( $\overline{\mathrm{SIR}})$ to mean the expected value of the $i$ th mobile received power over the expected value of the interference from the $K-1$ other mobiles and background noise. This is also the certainty-equivalent SIR and is given by

$\overline{\mathrm{SIR}}=\gamma_{i}^{c e}=\frac{E\left[G_{i i} F_{i i} P_{i}\right]}{E\left[\sum_{j \neq i} G_{i j} F_{i j} P_{j}+\sigma^{2}\right]}=\frac{G_{i i} P_{i}}{\sum_{j \neq i} G_{i j} P_{j}+\sigma^{2}}$.

As with [8], we also define the CEM (with noise) as the ratio of the certainty-equivalent SIR to the average SIR threshold,

$$
C E M_{i}^{\sigma}=\frac{\gamma_{i}^{c e}}{\gamma_{i}^{t h}}=\frac{G_{i i} P_{i}}{\gamma_{i}^{t h}\left\{\sum_{j \neq i} G_{i j} P_{j}+\sigma^{2}\right\}} .
$$

\section{Relation Between the $C E M^{\sigma}$ and Outage Probability}

Using the following result, we derive some bounds between the $C E M^{\sigma}$ and outage probability. For $z_{1}, \ldots, z_{n} \geq 0$ we have,

$$
1+k+\sum_{i}^{n} z_{i} \leq e^{k} \prod_{i}^{n}\left(1+z_{i}\right) \leq e^{k+\sum_{i}^{n} z_{i}}
$$

where $k$ is some constant.

From (4), we have

$$
\begin{aligned}
O_{i} & =1-\exp \left(\frac{-\sigma^{2} \gamma_{i}^{t h}}{G_{i i} P_{i}}\right) \prod_{j \neq i} \frac{1}{1+\frac{\gamma_{i}^{t h} G_{i j} P_{j}}{G_{i i} P_{i}}} \\
& =1-\frac{1}{\exp \left(\frac{\sigma^{2} \gamma_{i}^{t h}}{G_{i i} P_{i}}\right) \prod_{j \neq i} 1+\frac{\gamma_{i}^{t h} G_{i j} P_{j}}{G_{i i} P_{i}}} .
\end{aligned}
$$

Using the left-hand side inequality in (7),

$$
\begin{aligned}
O_{i} & \geq 1-\frac{1}{1+\frac{\sigma^{2} \gamma_{i}^{t h}}{G_{i i} P_{i}}+\sum_{j \neq i} \frac{\gamma_{i}^{t h} G_{i j} P_{j}}{G_{i i} P_{i}}} \\
& =1-\frac{1}{1+\frac{\gamma_{i}^{t h}\left\{\sum_{j \neq i} G_{i j} P_{j}+\sigma^{2}\right\}}{G_{i i} P_{i}}} \\
& =\frac{1}{1+C E M_{i}^{\sigma}} .
\end{aligned}
$$


Repeating the procedure above for the right-hand side inequality in (7),

$$
\begin{aligned}
O_{i} & \leq 1-\frac{1}{\exp \left(\frac{\sigma^{2} \gamma_{i}^{t h}}{G_{i i} P_{i}}+\sum_{j \neq i} \frac{\gamma_{i}^{t h} G_{i j} P_{j}}{G_{i i} P_{i}}\right)} \\
& =1-\frac{1}{\exp \left(\frac{\gamma_{i}^{t h}\left[\sum_{j \neq i} G_{i j} P_{j}+\sigma^{2}\right]}{G_{i i} P_{i}}\right)} \\
& =1-e^{-1 / C E M_{i}^{\sigma}} .
\end{aligned}
$$

The upper and lower bounds on outage are thus,

$$
\frac{1}{1+C E M_{i}^{\sigma}} \leq O_{i} \leq 1-e^{-1 / C E M_{i}^{\sigma}}
$$

which have the same form and tightness in the region of interest as the noiseless case in [8].

Note that $C E M_{i}^{\sigma}$ (or average SIR with a fixed $\gamma_{i}^{t h}$ ) and outage probability are inversely proportional. As we increase the $C E M_{i}^{\sigma}$ (or $\overline{\mathrm{SIR}}$ ), we get a lower outage probability and vice-versa.

\section{Power Control Algorithm}

\section{A. Problem Definition}

The aim of the PCA is to find the powers, $P_{i}$, and filter coefficients, $\mathbf{c}_{i}$ for $i=1, \ldots, K$, such that the total power transmitted by all users is minimized while all outage constraints are met. i.e., $O_{i} \leq O_{i}^{t}$. Stating this as an optimization problem, we have

$$
\begin{aligned}
& \min _{\mathbf{P}, \mathbf{c}} \sum_{i=1}^{K} P_{i} \\
& \text { s.t. } 1-e^{\frac{-\sigma^{2}\left(\mathbf{c}_{i}^{\top} \mathbf{c}_{i}\right) \gamma_{i}^{t h}}{G_{i i}\left(\mathbf{c}_{i}^{\top} \mathbf{s}_{i}\right)^{2} P_{i}}} \prod_{j \neq i} \frac{1}{1+\frac{\gamma_{i}^{t h} G_{i j}\left(\mathbf{c}_{i}^{\top} \mathbf{s}_{j}\right)^{2} P_{j}}{G_{i i}\left(\mathbf{c}_{i}^{\top} \mathbf{s}_{i}\right)^{2} P_{i}}} \leq O_{i}^{t}, \\
& \quad P_{i} \geq 0, \quad \mathbf{c}_{i} \in \mathbb{R}^{N} \quad i=1, \ldots, K
\end{aligned}
$$

where we used (4) with the receiver filter included and $O_{i}^{t}$ are target outage probability constraints. It can be shown that this problem is equivalent to the following, where the inner optimization has been inserted into the constraint set. See [17] for a similar refinement.

$$
\begin{aligned}
\min _{\mathbf{P}} & \sum_{i=1}^{K} P_{i} \\
\text { s.t. } & \min _{\mathbf{c}_{i} \in \mathbb{R}^{N}}\left\{1-e^{\frac{-\sigma^{2}\left(\mathbf{c}_{i}^{\top} \mathbf{c}_{i}\right) \gamma_{i}^{t h}}{G_{i i}\left(\mathbf{c}_{i}^{\top} \mathbf{s}_{i}\right)^{2} P_{i}}} \prod_{j \neq i} \frac{1}{1+\frac{\gamma_{i}^{t h} G_{i j}\left(\mathbf{c}_{i}^{\top} \mathbf{s}_{j}\right)^{2} P_{j}}{G_{i i}\left(\mathbf{c}_{i}^{\top} \mathbf{s}_{i}\right)^{2} P_{i}}}\right\} \leq O_{i}^{t}, \\
& P_{i} \geq 0
\end{aligned}
$$

\section{B. Optimal Power Control}

In this section we describe an iterative algorithm to solve the optimization problem (9).

Taking the log of the outage constraint from (9) and rearranging yields

$$
\frac{w_{i}+\frac{\tilde{G}_{i i} P_{i}}{\gamma_{i}^{t h}} \sum_{j \neq i} \log \left(1+\frac{\tilde{G}_{i j} P_{j}}{\frac{\tilde{G}_{i i} P_{i}}{\gamma_{i}^{t h}}}\right)}{\frac{\tilde{G}_{i i} P_{i}}{\gamma_{i}^{t h}}} \leq \log \left(\frac{1}{1-O_{i}^{t}}\right)
$$

with $w_{i}=\sigma^{2}\left(\mathbf{c}_{i}^{\top} \mathbf{c}_{i}\right), \tilde{G}_{i j}=G_{i j}\left(\mathbf{c}_{i}^{\top} \mathbf{s}_{j}\right)^{2}$, and finally

$$
P_{i} \geq \frac{w_{i}+\widehat{G}_{i i} P_{i} \sum_{j \neq i} \log \left(1+\frac{\tilde{G}_{i j} P_{j}}{\widehat{G}_{i i} P_{i}}\right)}{\widehat{G}_{i i} \log \left(\frac{1}{1-O_{i}^{t}}\right)}
$$

where $\widehat{G}_{i i}=\frac{\tilde{G}_{i i}}{\gamma_{i}^{t h}}$.

If we view (10) as representing a set of quasi-interference constraints on the power vector $\mathbf{P}$, we can define a new PCA where each user $i$ iteratively attempts to compensate for the interference. At convergence, we would like each of the outage constraints to be met.

We define

$$
\begin{aligned}
I_{i}\left(\mathbf{P}, \mathbf{c}_{i}\right) & =\frac{w_{i}+\widehat{G}_{i i} P_{i} \sum_{j \neq i} \log \left(1+\frac{\tilde{G}_{i j} P_{j}}{\widehat{G}_{i i} P_{i}}\right)}{\widehat{G}_{i i} \log \left(\frac{1}{1-O_{i}^{t}}\right)} \\
T_{i}(\mathbf{P}) & =\min _{\mathbf{c}_{i}} I_{i}\left(\mathbf{P}, \mathbf{c}_{i}\right)
\end{aligned}
$$

where we let $I_{i}\left(\mathbf{P}, \mathbf{c}_{i}\right)=P_{i}$ be (10) replaced with equality. We shall now refer to $I_{i}\left(\mathbf{P}, \mathbf{c}_{i}\right)$ as the interference function to maintain consistency with the framework in [13].

Furthermore, we propose the PCA

$$
\mathbf{P}(n+1)=\mathbf{T}(\mathbf{P}(n))
$$

where $\mathbf{T}(\mathbf{P})=\left[T_{1}(\mathbf{P}), \ldots, T_{K}(\mathbf{P})\right]^{\top}$ and is initialized with powers set to the receiver noise level $P_{i}(0)=\sigma^{2}, \forall i$ and matched filter coefficients $\mathbf{c}_{i}(0)=\mathbf{s}_{i}, \forall i$.

This PCA is similar in form to that in [17], however we are dealing with outage and average quantities on a slower time scale. In Section IV-C, we will see how a sub-optimal variant of (11)-(13) can be reduced to a PCA that is similar in form to [17].

Since our PCA (13) is of the standard form given by [13], Theorem 1 proposes that (12) is a standard interference function and thus the PCA converges to a fixed solution. The proof is based on the three properties of a standard interference function given in [13].

Theorem 1: $\mathbf{T}(\mathbf{P})$ is a standard interference function.

Proof: We recall that $G_{i j}>0, P_{i}>0$ and $0<O_{i}^{t}<1$. Thus for any fixed $\mathbf{c}_{i}, I_{i}\left(\mathbf{P}, \mathbf{c}_{i}\right)>0$. Therefore $T_{i}(\mathbf{P})=$ $\min _{\mathbf{c}_{i}} I_{i}\left(\mathbf{P}, \mathbf{c}_{i}\right)$ is positive and $\mathbf{T}(\mathbf{P})$ is also positive.

To prove monotonicity of (12), we will first show that (11) is monotonic for any fixed $\mathbf{c}_{i}$.

Note that $I_{i}\left(\mathbf{P}, \mathbf{c}_{i}\right)$ is monotonic in all $P_{j}, j \neq i$ - we only need to prove monotonicity for $P_{i}$. This is equivalent to proving monotonicity of

$$
y=x \log \left(1+\frac{k}{x}\right)
$$

where $x=\widehat{G}_{i i} P_{i}$ and $k=\tilde{G}_{i j} P_{j}$ is a constant.

It can be shown that (14) is monotonic for $k>0$. We have $k=\tilde{G}_{i j} P_{j}$ which is positive, thus (11) is monotonic. 
If $\mathbf{c}_{i}^{*}=\arg \min _{\mathbf{c}_{i}} I_{i}\left(\mathbf{P}, \mathbf{c}_{i}\right)$, then we have

$$
\begin{aligned}
T_{i}(\mathbf{P}) & =\min _{\mathbf{c}_{i}} I_{i}\left(\mathbf{P}, \mathbf{c}_{i}\right) \\
& =I_{i}\left(\mathbf{P}, \mathbf{c}_{i}^{*}\right) \\
& \geq I_{i}\left(\mathbf{P}^{\prime}, \mathbf{c}_{i}^{*}\right) \\
& \geq \min _{\mathbf{c}_{i}} I_{i}\left(\mathbf{P}^{\prime}, \mathbf{c}_{i}\right) \\
& =T_{i}\left(\mathbf{P}^{\prime}\right)
\end{aligned}
$$

Thus $T_{i}(\mathbf{P}) \geq T_{i}\left(\mathbf{P}^{\prime}\right)$ and (12) satisfies the monotonicity property.

To prove scalability, we note that for a fixed $\mathbf{c}_{i}$, we have

$$
\begin{aligned}
I_{i}\left(\alpha \mathbf{P}, \mathbf{c}_{i}\right) & =\frac{w_{i}+\alpha \widehat{G}_{i i} P_{i} \sum_{j \neq i} \log \left(1+\frac{\alpha \tilde{G}_{i j} P_{j}}{\alpha \widehat{G}_{i i} P_{i}}\right)}{\widehat{G}_{i i} \log \left(\frac{1}{1-O_{i}^{t}}\right)} \\
& <\alpha I_{i}\left(\mathbf{P}, \mathbf{c}_{i}\right)
\end{aligned}
$$

since the $\alpha$ 's cancel in the log term and the noise $w_{i}$ is nonzero.

If again $\mathbf{c}_{i}^{*}=\arg \min _{\mathbf{c}_{i}} I_{i}\left(\mathbf{P}, \mathbf{c}_{i}\right)$, then we have

$$
\begin{aligned}
\alpha T_{i}(\mathbf{P}) & =\min _{\mathbf{c}_{i}} \alpha I_{i}\left(\mathbf{P}, \mathbf{c}_{i}\right) \\
& =\alpha I_{i}\left(\mathbf{P}, \mathbf{c}_{i}^{*}\right) \\
& >I_{i}\left(\alpha \mathbf{P}, \mathbf{c}_{i}^{*}\right) \\
& \geq \min _{\mathbf{c}_{i}} I_{i}\left(\alpha \mathbf{P}, \mathbf{c}_{i}\right) \\
& =T_{i}(\alpha \mathbf{P})
\end{aligned}
$$

Thus $\alpha T_{i}(\mathbf{P})>T_{i}(\alpha \mathbf{P})$ and (12) satisfies the scalability property.

Since $\mathbf{T}(\mathbf{P})$ is a standard interference function, the PCA (11)-(13) converges to a final solution $\mathbf{P}^{*}=\mathbf{T}\left(\mathbf{P}^{*}\right)$. This solution is the minimum power required to meet all users' outage constraints. The filter coefficients converge to a new type of MUD, the Minimum Outage Probability (MOP) receiver.

\section{Average SIR and Outage Probability}

To meet our outage probability constraints, we require that $O_{i} \leq O_{i}^{t}$ for all $i$. Combining this inequality with the upper bound in (8), we can define a new constraint on $C E M_{i}^{\sigma}$ that when met, will guarantee that our original outage constraints are also met:

$$
O_{i} \leq 1-e^{-1 / C E M_{i}^{\sigma}} \leq O_{i}^{t} \quad i=1, \ldots, K .
$$

Rearranging the right-hand side of (15) yields,

$$
\begin{aligned}
e^{-1 / C E M_{i}^{\sigma}} & \geq 1-O_{i}^{t} \\
\frac{1}{C E M_{i}^{\sigma}} & \leq \log \left(\frac{1}{1-O_{i}^{t}}\right) \\
C E M_{i}^{\sigma} & \geq \frac{1}{\log \left(\frac{1}{1-O_{i}^{t}}\right)} \\
\gamma_{i} & \geq \frac{\gamma_{i}^{t h}}{\log \left(\frac{1}{1-O_{i}^{t}}\right)} \\
& =\Gamma_{i}^{t h}
\end{aligned}
$$

where we have used the definition of $C E M_{i}^{\sigma}$ from (6) and defined a new quantity,

$$
\Gamma_{i}^{t h}=\frac{\gamma_{i}^{t h}}{\log \left(\frac{1}{1-O_{i}^{t}}\right)}
$$

called the outage-mapped average SIR threshold. We can now define a new problem,

$$
\begin{array}{lll}
\min _{\mathbf{P}, \mathbf{c}} & \sum_{i=1}^{K} P_{i} & \\
\text { s.t. } & P_{i} \geq \frac{\Gamma_{i}^{t h}}{G_{i i}} \frac{\sum_{j \neq i} G_{i j}\left(\mathbf{c}_{i}^{\top} \mathbf{s}_{j}\right)^{2} P_{j}+\sigma^{2}\left(\mathbf{c}_{i}^{\top} \mathbf{c}_{i}\right)}{\left(\mathbf{c}_{i}^{\top} \mathbf{s}_{i}\right)^{2}} & \\
& P_{i} \geq 0, \mathbf{c}_{i} \in \mathbb{R}^{N} & i=1, \ldots, K
\end{array}
$$

where we have rearranged (5) to form a SIR constraint. This problem is mathematically equivalent to the power control problem in [17], however we consider average channel gains and the outage-mapped average SIR threshold $\Gamma_{i}^{t h}$ as parameters.

In a similar fashion to [17], the problem is equivalent to

$$
\begin{array}{ll}
\min _{\mathbf{P}} & \sum_{i=1}^{K} P_{i} \\
\text { s.t. } & P_{i} \geq \frac{\Gamma_{i}^{t h}}{G_{i i}} \min _{\mathbf{c}_{i} \in \mathbb{R}^{N}} \frac{\sum_{j \neq i} G_{i j}\left(\mathbf{c}_{i}^{\top} \mathbf{s}_{j}\right)^{2} P_{j}+\sigma^{2}\left(\mathbf{c}_{i}^{\top} \mathbf{c}_{i}\right)}{\left(\mathbf{c}_{i}^{\top} \mathbf{s}_{i}\right)^{2}} \\
& P_{i} \geq 0
\end{array}
$$

with an associated PCA given by,

$$
\begin{aligned}
\tilde{I}_{i}\left(\mathbf{P}, \mathbf{c}_{i}\right) & =\frac{\Gamma_{i}^{t h}}{G_{i i}} \frac{\sum_{j \neq i} G_{i j}\left(\mathbf{c}_{i}^{\top} \mathbf{s}_{j}\right)^{2} P_{j}+\sigma^{2}\left(\mathbf{c}_{i}^{\top} \mathbf{c}_{i}\right)}{\left(\mathbf{c}_{i}^{\top} \mathbf{s}_{i}\right)^{2}} \\
\tilde{T}_{i}(\mathbf{P}) & =\min _{\mathbf{c}_{i}} \tilde{I}_{i}\left(\mathbf{P}, \mathbf{c}_{i}\right) \\
\mathbf{P}(n+1) & =\tilde{\mathbf{T}}(\mathbf{P}(n))
\end{aligned}
$$

where $\tilde{\mathbf{T}}(\mathbf{P})=\left[\tilde{T}_{1}(\mathbf{P}), \ldots, \tilde{T}_{K}(\mathbf{P})\right]^{\top}$.

In [17], it was shown that the MMSE filter coefficients $\mathbf{c}_{i}$ minimize (17) and so we have the following iterative algorithm for the above problem:

$$
\begin{aligned}
\hat{\mathbf{c}}_{i} & =\frac{\sqrt{P_{i}}(n)}{1+P_{i}(n) \mathbf{s}_{i}^{\top} \mathbf{A}_{i}^{-1} \mathbf{s}_{i}} \mathbf{A}_{i}^{-1} \mathbf{s}_{i} \\
P_{i}(n+1) & =\frac{\Gamma_{i}^{t h}}{G_{i i}} \frac{\sum_{j \neq i} P_{j}(n) G_{i j}\left(\hat{\mathbf{c}}_{i}^{\top} \mathbf{s}_{j}\right)^{2}+\sigma^{2}\left(\hat{\mathbf{c}}_{i}^{\top} \hat{\mathbf{c}}_{i}\right)}{\left(\hat{\mathbf{c}}_{i}^{\top} \mathbf{s}_{j}\right)^{2}}
\end{aligned}
$$

where $\mathbf{A}_{i}=\sum_{j \neq i} P_{j} G_{i j} \mathbf{s}_{j} \mathbf{s}_{j}^{\top}+\sigma^{2} \mathbf{I}$ is updated on each iteration and the PCA is initialized with powers set to the noise level and matched filter coefficients. The convergence proof of this PCA mirrors that in [17].

Since we have used the upper bound on $O_{i}$ in the derivation above, the solution is sub-optimal, however we guarantee the outage constraints from the right-hand side of (15). Recall that in the region of interest, the outage bounds are tight, and so we expect that this PCA will result in nearly optimal performance as compared to the optimal MOP PCA.

\section{Simulation Results}

Our simulation considers a single circular CDMA cell with radius $1 \mathrm{~km}$. We assume uniform location of users within the cell who are subject to distance dependent loss (loss exponent 4) and log-normal (zero mean, $8 \mathrm{~dB}$ variance) shadowing. 


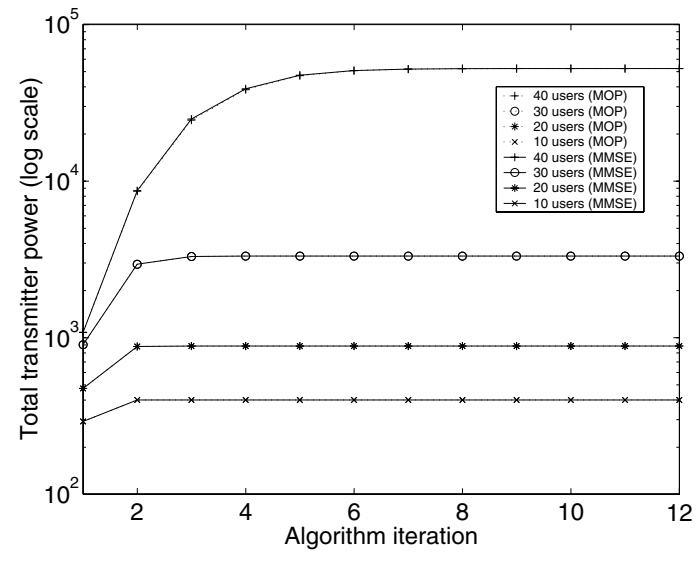

Fig. 1. Total transmitter power with 10-40 users.

A processing gain of 40 was chosen with AWGN power equal $\sigma^{2}=10^{-13}$, corresponding to approximately a $1 \mathrm{MHz}$ bandwidth.

We defined three classes of users, each having outage probability and SIR threshold pairs as $\{(5 \%, 10.53 \mathrm{~dB}),(10 \%$, $8.40 \mathrm{~dB}),(20 \%, 6.79 \mathrm{~dB})\}$. We assign $50 \%$ of users to the first class, $25 \%$ to the second and the remaining to the third.

User signature sequences were chosen randomly, and initial filter coefficients set to the matched filter. Powers were set to the noise power $\sigma^{2}=10^{-13}$ in preparation for the first iteration.

For $K=10,20,30,40$ users, Fig. 1 shows in log scale the sum power of all users as a function of the iteration step. The difference in powers between the optimal MOP PCA and MMSE PCA are almost indistinguishable, verifying earlier claims on the tightness of the bounds.

Figure 2 considers a system of 40 users. It shows the convergence of the outage probabilities for each user as a function of the iteration step. We clearly see the three outage probability classes $(5 \%, 10 \%$ and $20 \%)$ at convergence.

\section{CONCLUSION}

This paper introduced a new power control problem which aims to jointly optimize user powers and linear receiver filters according to outage constraints. An iterative algorithm to solve this problem was developed and convergence proved. A bound on the outage probability enabled a mapping to take place between outage and an average SIR threshold. From this, a sub-optimal PCA-MUD was developed that utilized the well known MMSE receiver. The approximation to the optimal solution was found to be exceptionally close in the $C E M^{\sigma}$ region of interest.

\section{REFERENCES}

[1] J. M. Aein, "Power balancing in system employing frequency reuse," COMSAT Technical Review, vol. 3, no. 2, pp. 277-300, 1973.

[2] R. W. Nettleton and H. Alavi, "Power control for a spread spectrum radio system," in IEEE Vehicular Technology Conference, pp. 242-246, 1983.

[3] J. Zander, "Performance of optimum transmitter power control in cellular radio systems," IEEE Transactions of Vehicular Technology, vol. 41, pp. 57-62, Feb. 1992.

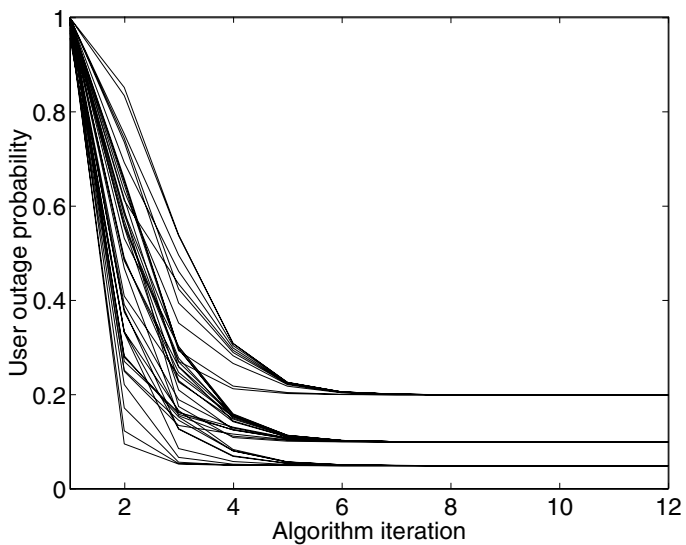

Fig. 2. Outage probability for MMSE PCA with 40 users.

[4] S. A. Grandhi, R. Vijayan, D. J. Goodman, and J. Zander, "Centralized power control in cellular radio systems," IEEE Transactions of Vehicular Technology, vol. 42, pp. 466-468, Nov. 1993.

[5] G. J. Foschini and Z. Miljanic, "A simple distributed autonomous power control algorithm and its convergence," IEEE Transactions of Vehicular Technology, pp. 541-646, 1993.

[6] S. V. Hanly, "An algorithm of combined cell-site selection and power control to maximize cellular spread spectrum capacity," IEEE Journal on Selected Areas in Communications, vol. 13, pp. 1332-1340, Sept. 1995.

[7] D. Julian, M. Chiang, and D. O'Neill, "Robust and QoS constrained optimization of power control in wireless cellular networks," in Vehicular Technology Conference, Atlantic City, NJ, USA, vol. 3, pp. 1932-1936, 2001.

[8] S. Kandukuri and S. Boyd, "Optimal power control in interferencelimited fading wireless channels with outage-probability specifications," IEEE Transactions on Wireless Communications, vol. 1, no. 1, pp. 46$55,2002$.

[9] N. Bui and S. Dey, "Optimal power control in CDMA over markov fading channels," in Proc. IEEE International Symposium on Information Theory, (Lausanne, Switzerland), p. 79, July 2002.

[10] R. D. Yates and C. Y. Huang, "Integrated power control and base station assignment," IEEE Transactions of Vehicular Technology, vol. 44, no. 3, pp. 638-644, 1995.

[11] S. Grandhi, J. Zander, and R. Yates, "Constrained power control," International Journal of Wireless Personal Communications, vol. 1, no. 4, 1995.

[12] S. Ulukus and R. D. Yates, "Stochastic power control for cellular radio systems," IEEE Transactions on Communications, vol. 46, pp. 784-798, June 1998.

[13] R. D. Yates, "A framework for uplink power control in cellular radio systems," IEEE Journal on Selected Areas in Communications, vol. 13 no. 7, pp. 1341-1347, 1995.

[14] S. Verdú, Multiuser detection. Cambridge, New York, USA: Cambridge University Press, 1998.

[15] S. Verdú, "Minimum probability of error for asynchronous gaussian multiple-access channels," IEEE Transactions of Information Theory, vol. 32, pp. 85-96, Jan. 1986.

[16] U. Madhow and M. L. Honig, "MMSE interference suppression for direct-sequence spread-spectrum CDMA," IEEE Transactions on Communications, vol. 42, no. 12, pp. 3178-3188, 1994.

[17] S. Ulukus and R. D. Yates, "Adaptive power control and MMSE interference suppression,” Wireless Networks, Special Issue on Multiuser Detection in Wireless Communications, vol. 4, no. 6, pp. 489-496, 1997.

[18] A. F. Almutairi, S. L. Miller, H. A. Latchman, and T. F. Wong, "Power control algorithm for MMSE receiver based CDMA systems," IEEE Communications Letters, vol. 4, no. 11, pp. 346-348, 2000.

[19] A. Yener, R. D. Yates, and S. Ulukus, "Interference management for CDMA systems through power control, multiuser detection, and beamforming," IEEE Transactions on Communications, vol. 49, pp. 1227 1239 , July 2001 